\title{
Espasmo coronario secundario a reacción alérgica 0 síndrome de Kounis. Caso clínico
}

\author{
Christian D auvergne ${ }^{1,2}$, Mario Araya ${ }^{1,2}$, \\ Alejandro Abufhele 1 . \\ Coronary spasm secondary to an \\ allergic reaction or Kounis syndrome. \\ Report of one case
}

We report a 79 year-old male with a history of an acute coronary syndrome subjected to coronary angioplasty and stent placement in the right coronary artery (RCA) four months ago. Since the patient continued with symptoms, a coronary angiography was performed. During the procedure, the patient developed a cutaneous allergic reaction and bronchospasm probably related to contrast media and a severe spasm of the RCA with inferior ST elevation in the absence of restenosis, that was difficult to treat. The presence of angina or an acute coronary syndrome and allergic reactions is known as the Kounis syndrome (Rev Méd Chile 2009; 137: 811-4).

(Key words: Coronary vasospasm; Drug hypersensitivity; Coronary angiography)

Recibido el 21 de octubre, 2008. Aprobado el 5 de diciembre, 2008.

${ }^{1}$ Departamento de Cardiología, Clínica Alemana de Santiago. ${ }^{2}$ Servicio Médico-Quirúrgico Cardiovascular, Instituto Nacional del Tórax. Santiago de Chile.

\begin{abstract}
I a base fisiopatológica que explica la aparición Lde un síndrome coronario agudo es en la mayoría de los casos el accidente de una placa aterosclerótica vulnerable y con claros signos inflamatorios o menos frecuentemente una erosión de una placa fibrocálcica, con la activación en ambas situaciones de las plaquetas y de la cascada de la coagulación, fenómeno denominado aterotrombosis.
\end{abstract}

$\overline{\text { Correspondencia a: Dr. Christian Dauvergne. Departamen- }}$ to de Cardiología, Clínica Alemana de Santiago. Vitacura 5051, Vitacura, Santiago. Teléfono 2101372.

E mail: cdauvergne@alemana.cl
Recientemente se ha descrito la asociación de reacciones alérgicas y síndromes coronarios agudos secundarios a vasoespasmos, lo que se conoce como síndrome de Kounis ${ }^{1}$, pudiendo éste variar desde la "angina alérgica" hasta llegar al "infarto agudo del miocardio alérgico". En su génesis se encuentran mediadores inflamatorios y son varias las causas reportadas como capaces de inducir este síndrome, entre las que se encuentran: numerosas drogas, reacciones de hipersensibilidad así como también exposición a agentes ambientales.

Describimos un caso de angina y vasoespasmo coronario desencadenado por una reacción alérgica, criterios que definen el síndrome de Kounis. 


\section{Caso ClínICO}

Hombre de 79 años de edad, con antecedentes de hipertensión arterial y tabaquismo detenido. No refiere antecedentes alérgicos. Inició hacía 4 meses una angina progresiva siendo sometido a una coronariografía, la que evidenció lesiones severas en el tercio proximal de la arteria coronaria derecha (CD) y otra en el tercio medio de la arteria descendente anterior (ADA). Se consideró la CD como el vaso responsable del cuadro y se sometió a una angioplastia con implante de un Stent Driver (Medtronic Inc.) en su segmento proximal. Por persistencia de angina de esfuerzo CF2 (CCS) que no respondió a tratamiento habitual ingresó a nuestra institución para un nuevo estudio angiográfico.

Se realizó una coronariografía por abordaje radial derecho $6 \mathrm{~F}$, previa administración de 50 ug iv de fentanylo y anestesia local con lidocaína. Se utilizó Iodixanol como medio de contraste intravascular. Inmediatamente posterior al estudio de la $\mathrm{CD}$, el que no mostró lesiones significativas ni reestenosis intrastent, presentó un rash cutáneo eritematoso papulomacular de distribución universal y broncoespasmo, por lo que se administraron

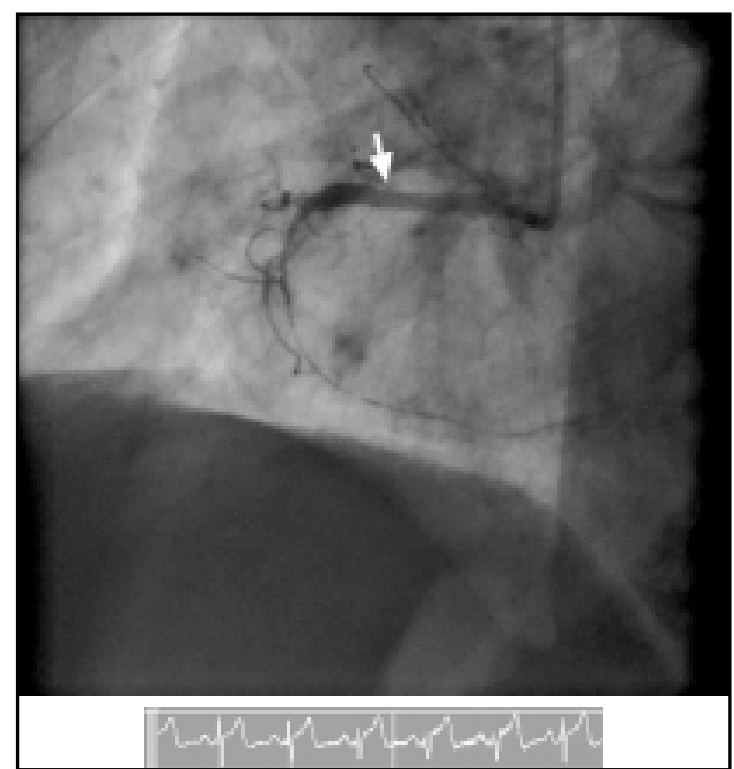

Figura 1. Nótese el espasmo difuso del vaso permaneciendo con su lumen real solo el segmento cubierto por el stent (flecha blanca). Abajo trazado del monito que muestra el supradesnivel ST. hidrocortisona $200 \mathrm{mg}$, clorfenamina $10 \mathrm{mg}$, salbutamol aerosol y oxigenoterapia. De inmediato inició dolor retroesternal intenso asociado a bradicardia e hipotensión, constatándose en el monitor un supradesnivel del segmento ST en derivaciones inferiores y bloqueo aurículo-ventricular completo. Se inyectó una vez más la CD observándose un espasmo difuso y severo que comprometía la arteria en toda su extensión (Figura 1), lo que se revirtió transitoriamente con el uso de nitroglicerina intracoronaria en bolo de 200 ug (Figura 2). El fenómeno se repitió en múltiples ocasiones, incluso luego de la administración coronaria de verapamilo en dosis de 200 a 400 ug y la infusión continua de nitroglicerina en dosis crecientes, hasta que se logró suprimir el espasmo.

Finalmente se logró realizar la angiografía de la ADA donde se constató una lesión moderada en el tercio medio y otra severa en el distal. Considerando una probable condición de hipersensibilidad como desencadenante del evento se realizó una angioplastia simple de la lesión distal y determinación de reserva de flujo fraccional (Pressure Wire. RADI Medical Systems AB)) en la lesión del tercio medio que fue no hemodinámicamente significativa (RFF 0,88).

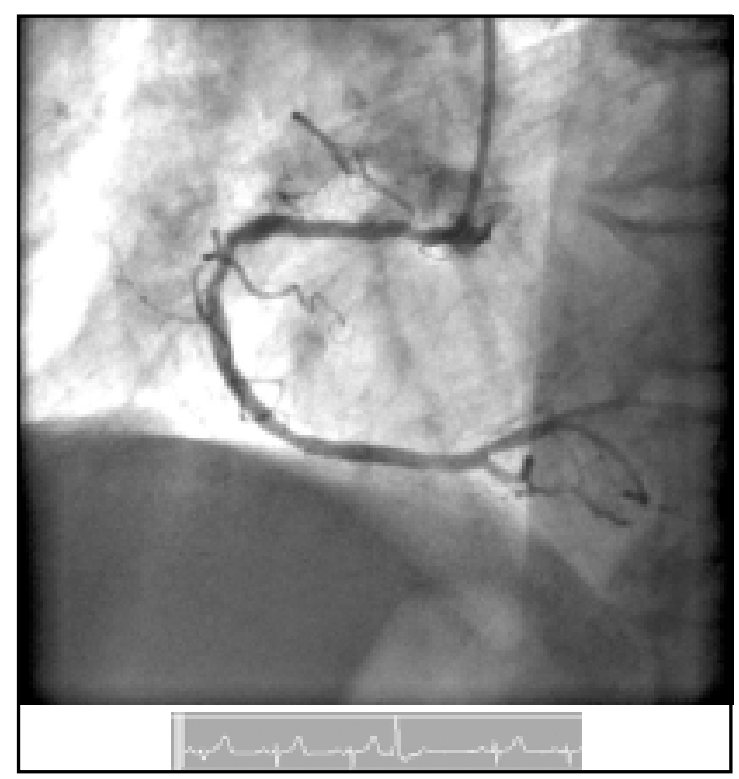

Figura 2. Regresión del espasmo del vaso. Abajo se observa en el trazado del monitor la desaparición del supradesnivel de ST. 
Se trasladó a la Unidad Coronaria donde se iniciaron antagonistas del calcio por vía oral y nitratos orales al día siguiente, aspirina, estatinas y loratadina; no presentó elevación de marcadores de injuria miocárdica. Fue dado de alta a los 3 días, manteniéndose asintomático en un seguimiento clínico de diez meses.

La asociación de hipersensibilidad y espasmo coronario severo en ausencia de historia previa de angina vasoespástica constituye como una unidad diagnóstica el síndrome de Kounis ${ }^{1}$.

\section{DisCuSIÓN}

Se ha considerado que el vasoespasmo puede ser secundario a una hiperreactividad arterial causada por inflamación local en la pared de la arteria coronaria. Forman et $\mathrm{al}^{2}$ reportaron un paciente con una angina vasoespástica complicada con una muerte súbita en quien se documentó la infiltración de mastocitos en el sitio de documentación angiográfica del espasmo coronario. También Kochi et $\mathrm{al}^{3}$ reportaron la infiltración local de células inflamatorias en la adventicia de pacientes con angina vasoespástica. Kovanen et al encontraron, en autopsias de pacientes fallecidos por infarto agudo del miocardio, una concentración 200 veces superior de mastocitos en el sitio de ruptura de una placa coronaria que en los indemnes ${ }^{4}$. Takagi et al reportaron cuatro casos de angina vasoespástica refractaria a tratamiento que fueron eficazmente resueltas con corticoesteroides 5 .

Durante procesos alérgicos agudos existe liberación de productos desde los mastocitos que incluyen histamina, metabolitos del ácido araquidónico tales como prostaglandinas y leucotrienos, y factor de agregación plaquetaria los cuales pueden causar contracción del músculo liso ${ }^{6}$.

Kounis describe la asociación de alergia y espasmo coronario, siendo éste la concurrencia de un síndrome coronario agudo con reacciones alérgicas o de hipersensibilidad y anafilácticas o anafilactoides ${ }^{1,7}$. Han sido descritas dos variantes del síndrome: el tipo I se presenta en pacientes con arterias coronarias normales y el tipo II en la que existe enfermedad ateromatosa en las que las reacciones de hipersensibilidad pueden no sólo inducir espasmo sino además erosión y rotura de la placa con la consiguiente trombosis coronaria subsecuente . $^{\text {. }}$.

Las causas del síndrome de Kounis incluyen ${ }^{1,7}$ : agentes ambientales, variados fármacos de distintas clases y sustancias como el látex, medio de contraste, desinfectantes cutáneos etc. (Tabla 1).

Es importante hacer notar que los metales componentes de los stent como el níquel, cromo y molibdeno pueden generar reacciones alérgicas e

Tabla 1. Causas de síndrome de Kounis

\begin{tabular}{|lll|}
\hline Condiciones clínicas & Fármacos & Agentes ambientales \\
\hline Angio-edema & Antibióticos & Mordedura de hormiga \\
Asma bronquial & Analgésicos & Picadura de abeja \\
Anafilaxia inducida por ejercicio & Antineoplásicos & Picadura de avispa \\
Alergia a alimentos & Medios de contraste & Medusas \\
Mastocitosis & Anestésicos intravenosos & Venenos \\
Enfermedad del suero & Antiinflamatorios no esteroidales (AINES) & Latex \\
Urticaria & Desinfectantes de piel & \\
& Corticosteroides & Ingestión de moluscos \\
& Trombolíticos & Veneno de víbora \\
\hline
\end{tabular}

Adaptado de Kounis NG. Kounis syndrome (allergic angina and allergic myocardial infarction): A natural paradigm? Int J Cardiol 2006; 110: 7-14. 
inflamatorias locales. Las reacciones de hipersensibilidad al níquel ocurren sobre $17 \%$ de la población. La alergia local seguida de manifestaciones sistémicas ha requerido la remoción de dispositivos intracardíacos ${ }^{8}$. En la actualidad está bien establecido que los stent liberadores de fármacos se asocian a reacciones de hipersensibilidad. Datos de la FDA reportan 262 casos de hipersensibilidad después de la implantación de estos dispositivos, y de éstos 17 se relacionaron directamente con los mismos; esta reacción se asoció a trombosis tardía fatal en 4 de los enfermos, demostrándose infiltración eosinofílica en la necropsia?.

No existe tratamiento bien establecido al momento, sobre todo qué terapias habituales usadas para el manejo de reacción de hipersensibilidad como los corticosteroides incluso pueden inducir el síndrome. Pero intentos se han hecho para contrarrestar los efectos de los mediadores de inflamación con antagonistas, inhibidores de la biosíntesis y bloqueadores de receptores de los mismos ${ }^{1}$.

En nuestro caso claramente el fenómeno vasoespástico es desencadenado por una reacción de

\section{REFERENCIAS}

1. Kounis NG. Kounis syndrome (allergic angina and allergic myocardial infarction): A natural paradigm? Int J Cardiol 2006; 110: 7-14.

2. Forman MB, OAtes JA, Robertson D, Roberts LJ, VIRMANI R. Increased adventitial mast cells in a patient with coronary spasm. N Engl J Med 1985; 313: 1138-41.

3. Kochi K, Takebayashi S, Hiroki T, Nobuyoshi $M$. Significance of adventitial inflammation of coronary artery in patients with unstable angina: Results at autopsy. Circulation 1985; 71: 709-16.

4. Kovanen PT, KaArtinen M, PaAvonen T. Infiltrates of activated mast cells at the site of coronary atheromatous erosion or rupture in myocardial infarction. Circulation 1995; 92: 1084-8.

5. Takagi S, Goto Y, Hirose e, Terashima M, Sakuragi $\mathrm{S}$, SuZUKI S ET AL. Successful treatment of refractory hipersensibilidad, si bien uno pudiera atribuirlo sólo al medio de contraste (Iodixanol), es interesante el hecho de que solo la coronaria derecha haya experimentado el espasmo severo y subintrante. Así uno podría especular que existió además una reacción alérgica e inflamatoria local en esta arteria, lo que podría estar en relación al stent implantado tres meses antes en cuya composición está el níquel. Por lo tanto podemos suponer que asistimos a una reacción de hipersensibilidad tanto local como sistémica en su origen que se manifestó durante el estudio angiográfico.

En suma, comunicamos el caso de un vasoespasmo coronario severo desencadenado por una reacción de hipersensibilidad, constituyéndose el diagnóstico de síndrome de Kounis. Dado que nuestro enfermo tiene enfermedad coronaria documentada representa el tipo II de la entidad. Este síndrome, probablemente subdiagnosticado, debe considerarse como potencial mecanismo fisiopatológico en algún subgrupo de pacientes los que probablemente requieran un enfoque terapéutico particular. vasospastic angina with corticosteroids. Coronary arterial hyperreactivity caused by local inflammation? Cir J 2004; 68: 17-22.

6. Kounis NG, Zavras GM. Allergic angina and allergic myocardial infarction. Circulation 1996; 94: 1789.

7. Kounis NG, Hahalis G, Theoharides TC. Coronary stents, hypersensitivity reactions, and the Kounis Syndrome. J Interven Cardiol 2007; 20: 314-23.

8. Dasika UK, Kanter KR, Vincent R. Nickel allergy to percutaneous patent foramen ovale occlude and subsequent systemic nickel allergy. J Thoracic Cardiovasc Surg 2003; 125: 2112-13.

9. Nebeker JR, Virmani R, Bennett CL, Hoffman JM, Samone MH, Alvarez J et al. Hypersensitivity cases associated with Drug-Eluting Coronary Stents. A review of available cases from the research on Adverse Drug Event and Reports (RADAR) Project. J Am Coll Cardiol 2006; 47: 175-81. 